\title{
Deployment Scheme in Wireless Sensor Network: A Review
}

\author{
Avantika Thakur \\ M.Tech(Scholar) \\ CGC, Landran
}

\author{
Devendra Prasad, PhD \\ Professor \\ CGC, Landran
}

\author{
Amit Verma, PhD \\ Professor \\ CGC,Landran
}

\begin{abstract}
Nowadays Wireless sensor networks (WSNs) being applied in many different civilian applications like vehicle tracking, habitat monitoring, forest surveillance, earthquake observation, biomedical or health care requests and building surveillance. To a large extent the efficiency of WSNs depends on the coverage provided by the deployment scheme. Different deployment demands and optimization goals are required in different environment. This study keep focus on coverage, connectivity and energy efficiency which have great impact on the performance of WSNs. Node deployment is a fundamental problem to be solved in WSNs. An appropriate node deployment scheme can reduce the issues in WSNs. Further it can extend the lifetime of WSNs by minimizing energy consumption. This paper investigated some deployment schemes, analysed and identifying their requirement.
\end{abstract}

\section{Keywords}

Challenges in WSN, Node deployment, Energy efficiency, BS.

\section{INTRODUCTION}

A wireless sensor network (WSN) usually consists of a large number of battery-powered sensor nodes ( $\mathrm{SNs}$ ). These $\mathrm{SNs}$ are small wireless devices with constrained in terms of energy, communication and computational resources. In WSNs energy-efficient medium-access control (MAC) is designed for minimizing energy consumption while achieving the endto end delay constraint to meet applications' requirements. WSNs are becoming an active area of research, where SNs are equipped with sensing, processing, and wireless networking capability. They can automatically collect the data and report the quantities to the sink. Recently, many WSNs have been designed and deployed for various applications. WSNs are used in a wide range of potential applications together with military, medical, and robotic, which explains the significant attention drawn by these types of networks in research field. SNs are usually battery powered, conserving their energy and prolonging the network life time are prime goals while designing protocols for WSNs. WSN consists of large number of sensing nodes which helps in observing the physical and environmental conditions such as temperature sound, pressure etc. to obtain the information from the atmosphere [1].

A recent emerged is a new research topic in wireless ad-hoc sensor network. WSN have long-term ability to transform our lives, economic potential, various novel system constructing challenges. SNs also position number of novel concept and reduce the issues. Such as position, deployment, fundamental problems and tracking, in that several application rely on them for required information [2].The general term is coverage, reply the questions about quality of service that could be given by a particular SN. An integration of numerous kinds of sensors like acoustic, optical and seismic etc. in individual platform network and analysis of the over-all coverage of the system also presents numerous challenges.

Rest of the paper is organised as follows, Section 2 describes the classification of node deployment, Section 3 describes the deployment scheme in wireless sensor network Section 4 describes the performance index of node deployment and problem definition is described in section 5. And finally a conclusion is made in section 6 .

\section{CLASSIFICATIONOFDEPLOYMENT SCHEMES}

The researches presently concentrated on static and the dynamic deployment scheme for the placement of SNs in the deployment area.

The static deployment chooses the best location for SNs, and the position of the SNs is not changed throughout the lifetime of WSNs. At present, the static deployment includes the deterministic deployment and the random deployment. The deterministic deployment schemes initially analysed deployment area and then carries deployment. The study of deterministic SN deployment based on the target coverage is presented in [7]

In order to make the WSNs get the maximum performance, SNs need automatically move to proper location, and then start to work. In random deployment, SNs are thrown randomly and then using a variety of optimization algorithm such as virtual force algorithm, virtual force oriented particles algorithm, simulated annealing algorithm, particle swarm optimization algorithm and simulated annealing genetic algorithm to find the optimal position for SNs [6]

\section{DEPLOYEMENT SCHEMES IN WIRELESS SENSOR NETWORK}

In WSNs, main challenges are the deployment of the sensor nodes in the deployment region to satisfy incessant sensing with extended network life-time and uniform coverage area. Several architectures and node deployment methods have been developed for WSN, depending up-on the need of application. We focus our study on followings deployment methods for WSNs, random deployment, grid deployment, group-based deployment and grid group deployment [3]. 


\subsection{Random Deployment}

In random deployment schemes SNs are randomly dropped from air. On the other hand, in planned deployment, SNs are set at exact locations one-by-one according to their communication range. Normally, the positions are choosing to optimise the number of SNs. Random deployment is most suited for unreachable, hazardous or large scale open environments. However, planned deployment technique is time consuming but costing the less number of SNs. [9]

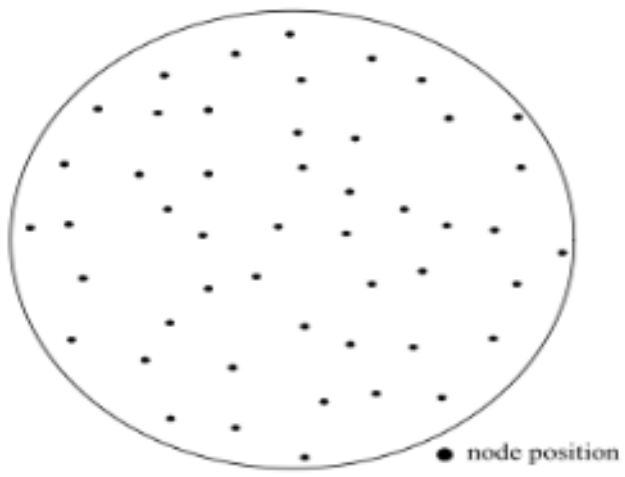

Figure 1: Randomly deployed SNs[9]

\subsection{Grid Deployment}

As excess idleness is required to overcome uncertainty it could be very expensive. Grid deployment is a striking approach for moderate to large-scale coverage-oriented deployment due to its simplicity and scalability. Grid deployment is conducted by dropping sensors row-by row using a moving carrier. Previously many studies have explored the belongings of grid deployment in the ideal situation where individual sensors are placed exactly at grid points. However, in practice, it is often infeasible to guarantee exact placement due to various errors including misalignments and random misplacement [4].

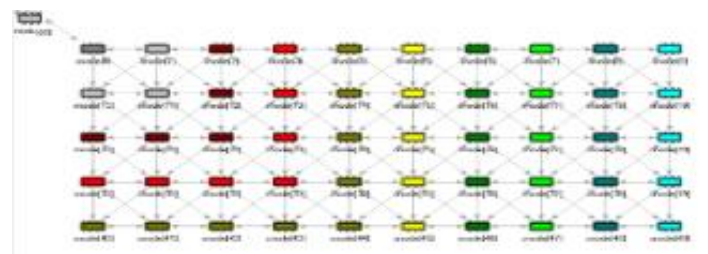

Figure 2: Grid deployment[4]

There are three popular grid layouts namely, unit square, an equilateral triangle, a hexagon, etc. Among them, we examine a square grid because of its natural placement strategy over a unit square. A grid deployment is considered a good deployment in WSN, especially for the coverage performance.

\subsection{Group Based Deployment}

In this SNs are deployed in groups and after deployment SNs closer together from a group. The group deployment may be used in order to improve the coverage of the target region and provides more control over the corporal distribution of SNs and also a convenient way of carrying out the deployment. However, the main motivation for considering group deployment is that, it provides partial positioning knowledge that can be used in order to improve the connectivity of the network. [8]

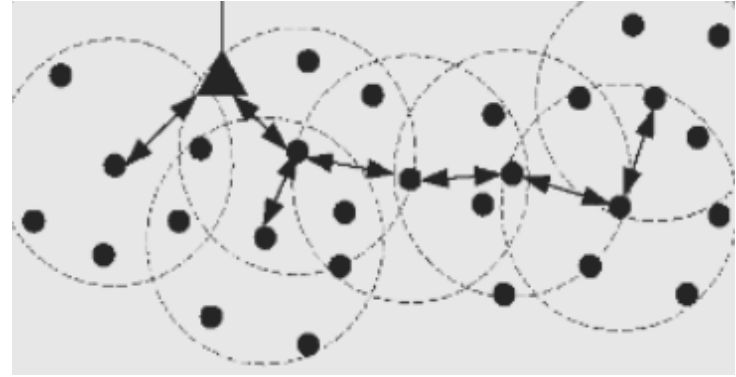

Figure 3: Group based deployment

\subsection{Grid Group Deployment}

The Grid-group deployment scheme for WSNs is discussed in [6].When SNs are deployed in a region; they need not communicate with all other SNs due to limited power. So the entire region is divided into equal-sized squares or grids.

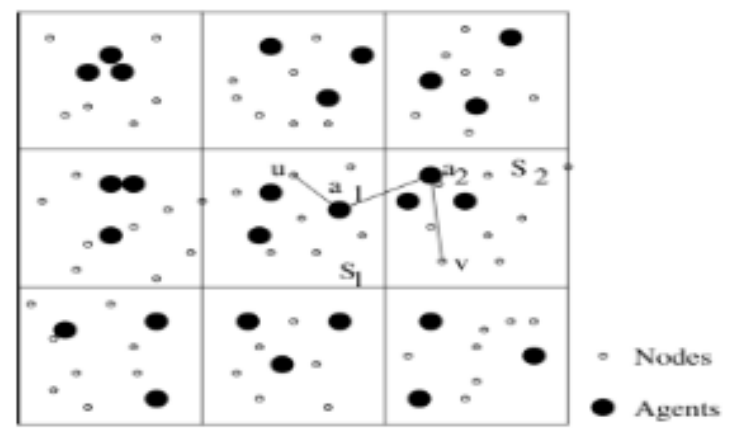

Figure 4: Grid group Deployment

This scheme has advantage that all SNs within a particular group can communicate with each other directly and they can communicate with SNs in other region via special nodes calledagentwhich have more resources than the general nodes as shown in Figure 4. It is also assumed that it is more difficult to compromise an agent as compared to a normal SN. The number of agents in a section is always three irrespective of the size of network.This structure ensures that even if one region is totally disconnected it is not affected.It is assumed that grid-group deployment is a very good resiliency in terms of the fraction of nodes detached and regions disconnected [5].

The reliability of WSNs can be measured on its ability to maintain the network connectivity after the occurrence of fault and re-establishing the network by reconfiguring it. In[7] authorsproposed a scheme to detect the network partition and network connectivity.In this various models have been proposed to deploy SNs in large-scale open regions. This work made use of MMUs that place themselves on appropriate locations provided by the Base station. The proposed system enhances the life time of WMNs and reconfigures the network.

In [8] authors proposed a Neighbour Assisted Deployment Scheme (NADS) to uniformly distribute the erratically blowout Mobile Sensor Nodes (MSNs) within a candidate region. Entire candidate region is divided into Square SubRegions (SSRs) which are further separated into regular hexagons and center of these hexagons forms the Desired Locations (DLs) for placement of MSNs. NADS uses 3-phase incremental approach for optimal placement of MSNs. The result of each phase forms the organization to assist the placement of outstanding unplaced MSNs. In each phase MSNs communicate locally with each other to elect the most 
appropriate one among them to relocate to the nearest DL. Factors such as dropping height, wind speed, parachute size, battery size and MSN weight are considered to determine the area approachable by any released MSN. This scheme limits the number of additions performed at each MSN, regardless of the size of the candidate region. This scheme is realistic, ascendable and yields better performance in terms of coverage, connectivity, setup-time and energy-efficiency with minimum number of MSNs as compared to existing schemes.

Authors in [9] presented a scheme for homogeneous distribution of randomly distributed mobile sensor nodes (MSNs) in the deployment area. The deployment area is square in shape, which is separated into number of concentric regions centred at Base Station, these regions are separated by half of the communication range and further deployment area is divided in to numbers of steady hexagons. To realise the maximum coverage and better connectivity MSNs sets themselves at the center of the hexagon on the guidance provided by the BS which is positioned at one of the corner in the deployment area. The simulation results shows that the presented scheme is better than some existing schemes in terms of number of MSNs compulsory for same coverage area and average movement compulsory by MSNs to fix themselves at the desired location and energy efficiency.

\section{PERFORMANCE INDEX OF NODE DEPLOYMENT SCHEMES}

The main purposes of deployment schemes are to increase the reporting area, enhance network connectivity, prolong the network lifetime, make the load balance, improve the accuracy of the data transmission and reinforce the tolerance of SNs. Itis not feasible to achieveall these by using any single deployment scheme. Even if it meets all major and minor needs the deployment cost needed to be considered. Generally, the optimization of the SNs deployment mainly includes the following performance indexes [10].

\subsection{Coverage Area}

To get maximum coverage is always the hotspot of the optimization problem in the deployment of SNs in WSNs. Coverage is an imperative issue and is related to energy saving, connectivity, and network reconfiguration. It mainly concern with how to deploy the SN to achieveactive coverage of the service-area so that every point in the service-area is monitored by at least one SN. A good coverage is indispensable for the effectiveness of WSNs.

In [11] the coverage area is assumed to be of a disk shape and the transmission range of SNs are equal to the radius of the disk shape deployment area, and the ratio of the area covered by node against whole area of deployment is the index of the monitoring coverage area.

In [12] a kind of model similar to grid scanning algorithm, where the obstacle is hinder the uncovering of SNs to the target and the accuracy of the target detection probability stands changing with the distance between SNs and detection target. It suggests a method of using the smallest quantity of SNs to realize the maximum attention and presents the triangle grid computing algorithm where any three adjacent nodes form an equilateral triangle.

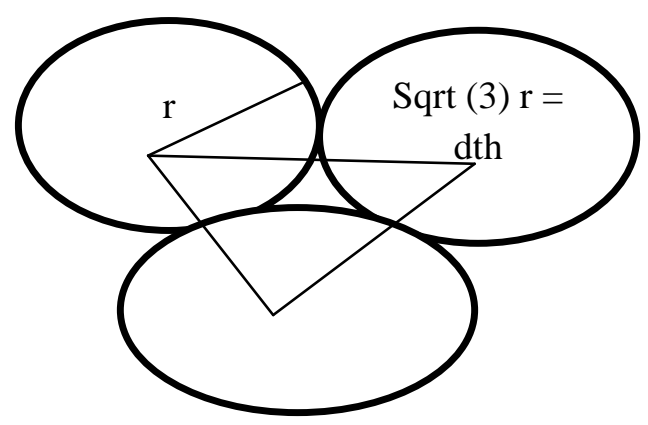

Figure 5: Maximum Coverage Area[12]

\subsection{Net Connectivity}

In WSN connectivity deals with the communication between, SN and BS, BS and the client, client and server. Earlier the network connectivity is not difficult problem. It considered the complete coverage and connectivity of the SNs, which are located within the identified radius. Due to whichonly routing between SNs and BS is considered to send the data [13].

\subsection{Network Lifetime}

One of the most important requirements of WSN is to optimise the energy consumption. Hence, there is a need for energy efficient communication and routing techniques that increase the network lifetime. The major cause of energy waste is collision. When a node receives more than one packet at the same time, these packets are termed collided, even when they coincide only incompletely. All containers that cause the collision have to be discarded and retransmissions of these packets are required, which increases energy consumption.The second reason for energy waste is over-hearing, which means that a node receives packets that are destined to other nodes. The third energy waste occurs as a result of control-packet over-head. In [14] authors investigate differences of the behaviour of agent based protocols in real deployment associated to the outcomes produced using custom based simulator, which ignores the lower layers effects, such as packet collision and overhearing.

\section{PROBLEM DEFINATION}

Network screen is a situation in which network is divided into numerous small sub networks due to certain difficulties caused by climatic change or disaster. The Problem is divided into two main parts [15].

\subsection{Network partition occurrence detection}

Since sensor nodes in adversely affected regions goes out of service and no longer able to maintain the connectivity of network. The location of division, shape of partition and size of the partitions need to be detected by Base Station to take appropriate actions to re-connect the partitioned networks. Various methods may be used for determining the likelihood of occurrence of partition, among those weather information provided by satellite can be most helpful.Base Station checks the current endure report generated by satellites on regular interval to keep track of weather conditions viz. rainfall, tornado, earth quake etc. in the deployment area. When critical weather conditions are sensed by Base Station it starts the system inspection process to determine partitions, if occurred. Based on the result obtained after network inspection, Base Station came to know about the occurrence of partition. 


\subsection{Determining number of partitions}

Initially, multipurpose mobile units are inactive and come in to action when invoked by Base Station. If occurrence of partitions is detected by BS it initiates all the MMUs to achieve the same inspection process to determine the size and location of partition to which they belong. The results of inspection are sent to BS directly by MMUs by temporarily increasing their communication range.

In our research work we will improve the network life, optimize the delay and overload using optimization techniques.

\section{CONCLUSION}

In this paper we discussed about the various deployment schemes but mainly focus on coverage connectivity and energy efficiency .The performance of WSN is greatly affected by these parameters. These parameters must be considered during the deployment of WSN. An efficient deployment scheme can reduce these issues in WSN up to large extent .This paper investigate some of the deployment schemes, analyse them and identify their requirements.

\section{REFERENCES}

[1]. Zhang, Haitao, and Cuiping Liu. "A review on node deployment of wireless sensor network." International Journal of Computer Science Issues(IJCSI), vol. 9, no. 3, pp 378-383,Nov.(2012).

[2]. M. Chithik Raja, and V. S. Balasubramanian, "Analysis and a report of wireless sensor networks and applications", International Journal of computer science issues, Vol. 8, No. 4, pp. 589-597,July 2011.

[3]. Vikrant, Sharma. "Policy for planned placement of sensor nodes in large scale wireless sensor network." KSII Transactions on Internet \& Information Systems Vol.,10, no. 7 ,pp.3213-3230 july(2016).

[4]. Matt Welsh, Dan Myung, Mark Gaynor, and Steve Moulton. "Resuscitation monitoring with a wireless sensor network". In Supplement to Circulation: Journal of the American Heart Association,October 2003.

[5]. Alan Mainwaring, Joseph Polastre, Robert Szewczyk, and David Culler. "Wireless sensor networks for habitat monitoring", In First ACM International Workshop on Wireless Sensor Networks and Applications, 2002.

[6]. X. He, X. L. Gui, and J. An, "A Deterministic Deployment Approach of Nodes in Wireless Sensor Networks for Target Coverage", Journal of XiAnJiaotong University Vol. 44, No.6, pp.6$10,2010$.
[7]. Sharma, Vikrant, R. B. Patel, H. S. Bhadauria, and Devendra Prasad. "NADS: Neighbor Assisted Deployment Scheme for Optimal Placement of Sensor Nodes to Achieve Blanket Coverage in Wireless Sensor Network." Wireless Personal CommunicationsVol. 90, no. 4,pp-1903-1933, june (2016)

[8]. Sharma, Vikrant, R. B. Patel, H. S. Bhadauria, and D. Prasad. "Deployment schemes in wireless sensor network to achieve blanket coverage in large-scale open area: A review." Egyptian Informatics Journal vol.17, no. 1 ,pp.45-56,august (2015)

[9]. Kumar, Ajay, Vikrant Sharma, and D. Prasad. "Distributed Deployment Scheme for Homogeneous Distribution of Randomly Deployed Mobile Sensor Nodes in Wireless Sensor Network." Editorial Preface Vol.4, no. 4,pp.139-146, (2013).

[10].Beutel, Jan, Kay Römer, Matthias Ringwald, and Matthias Woehrle. "Deployment techniques for sensor networks." In Sensor Networks, Springer Berlin Heidelberg, pp. 219-248, ( 2010).

[11].Kumar, Rahul, and SumanDeswal. "A Review paper on improving power consumption in Wireless Sensor Network." International Journal of Science, Engineering and Technology Research (IJSETR), Vol. 4, Issue 7, pp.2390-2396,July (2015).

[12].Haule, Joseph, and Kisangiri Michael. "Deployment of wireless sensor networks (WSN) in automated irrigation management and scheduling systems: a review." In Science, Computing and Telecommunications (PACT), 2014 Pan African Conference on, pp. 86-91. IEEE, july(2014).

[13].Al-Obaisat, Yazeed, and Robin Braun. "On wireless sensor networks: architectures, protocols, applications, and management." In International Conference on Wireless Broadband and Ultra Wideband Communication (AusWireless),vol.35,. pp.1115,(2012).

[14].Aghajan, Hamid, and Andrea Cavallaro, eds. Multicamera networks: principles and applications. Academic press, pp.3-27,(2009).

[15].Sahni, Sartaj, and XiaochunXu. "Algorithms for wireless sensor networks." International journal of distributed sensor networks vol.1, no. 1,pp.35-56,sep (2005). 